\title{
VACINAL COVERAGE OF PATIENTS WITH RHEUMATOID ARTHRITIS OF BRASILIA COHORT
}

Alex Luiz Pires (Hospital Universitário de Brasíla, Brasília, DF, Brasil), Talita Yokoy (Hospital Universitário de Brasília, Brasília, DF, Brasil), Isabela Sousa Russo (Hospital Universitário de Brasília, Brasília, DF, Brasil), Tassiane Raquel Morais (Hospital Universitário de Brasília, Brasília, DF, Brasil), Ana Paula Gomides (Hospital Universitário de Brasília, Brasília, DF, Brasil), Licia Maria Henrique Mota (Hospital Universitário de Brasília, Brasília, DF, Brasil)

\section{BACKGROUND}

Rheumatoid arthritis (RA) is an autoimmune inflammatory systemic disease. Infections are an important cause of morbidity and mortality in patients with RA. It is estimated that these patients have a double risk of developing infectious conditions when compared to healthy patients of the same sex and age; vaccination is the preventive measure with the greatest impact on the control of infectious diseases and their complications. The objective of this study is to evaluate the vaccination coverage of patients with RA from the Brasilia cohort 5 years after the establishment of the vaccine verification protocol by the medical team of the service.

\section{MATERIALS AND METHODS}

Observational study with a cross-sectional design. Data collected by an evaluator external to the assistant team by means of a proper questionnaire and check of the vaccination card.

\section{RESULTS}

71 patients were evaluated. $88.7 \%$ women, mean age of 53.6 years ( \pm 13.3 years). Mean diagnostic time of 8 years ( \pm 4.8 years), $83.1 \%$ of patients were in remission at the time of the interview and the use of MTX in monotherapy was observed in $23.9 \%$ of these patients. Compared to the evaluation of the 2012 vaccine coverage with 68 patients, patients are more oriented by physicians regarding the use of vaccines $(72,9 \%$ vs. $7.3 \%)$ increased the use of inactive or recombinant vaccine $(97,4 \%$ vs $84 \%)$ and there was a slight decrease in the use of vaccine with attenuated live component ( $45 \%$ vs. $47 \%)$. Of the patients who used vaccines with live component, more than half $(68,7 \%)$ were immunized before the diagnosis of RA. Patients who used a vaccine with a live or attenuated component after the diagnosis of RA, did so without the recommendation of rheumatologists at the service. The sources of information on vaccination were researched. The media and doctors of the service are the main sources of information in $58,6 \%$ and $30 \%$ of the cases, respectively. In addition, $87,1 \%$ are not afraid to receive vaccines and 3 patients reported some side effect attributed to the vaccine (but no health professional confirmed such a relationship).

\section{CONCLUSION}

There was an improvement in vaccination coverage with dead viruses, especially influenza and pneumonia, due to systematic check of the vaccination card and the recommendations of the 2012 Consensus of the Brazilian Society of Rheumatology on Vaccination in Patients with RA. 